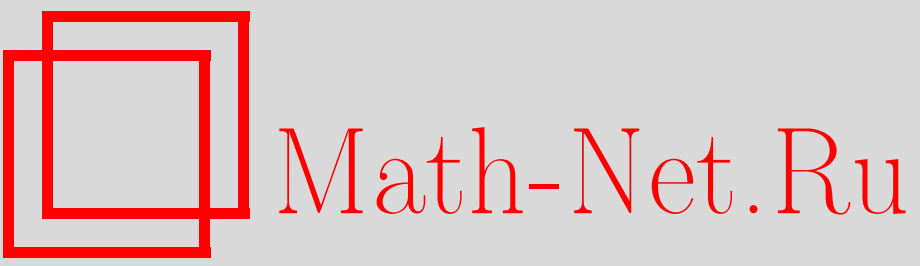

А. В. Булинский, С. В. Дильман, Универсальная нормировка в законе повторного логарифма, УМH, 2002, том 57, выпуск 2, 193-194

DOI: https://doi.org/10.4213/rm504

Использование Общероссийского математического портала Math-Net.Ru подразумевает, что вы прочитали и согласны с пользовательским соглашением

http://www.mathnet.ru/rus/agreement

Параметры загрузки:

IP: 44.207 .124 .84

26 апреля 2023 г., 11:46:28 


\title{
УНИВЕРСАЛЬНАЯ НОРМИРОВКА В ЗАКОНЕ ПОВТОРНОГО ЛОГАРИФМА
}

\author{
А. В. Булинский, С. В. ДильмАн
}

Классический закон повторного логарифма, впервые установленный для сумм независимшх бернуллиевских величин Хинчиным, является источником различных обобщений. Так, известная теорема Хартмана-Винтнера утверждает, что для независимых одинаково распределенных величин $X_{1}, X_{2}, \ldots$ с $\mathrm{E} X_{1}=0$ и $\mathrm{E} X_{1}^{2}=1$ (такую последовательность будем назьвать стандартной) верхний и нижний пределы нормированных сумм $S_{n} / \sqrt{2 n \ln \ln n}$ равны п.н. соответственно 1 и -1 ; здесь $S_{n}=X_{1}+\cdots+X_{n}, n \geqslant 3$. После доказательства знаменитого интегрального критерия Колмогорова-Петровского, описывающего "верхние" и "нижние" функции для броуновского движения, аналогичный критерий в схеме суммирования независимых случайных величин был получен Эрдёшем и Феллером. Если этому критерию удовлетворяет последовательность центрированных величин $X_{n}$ (возможно, зависимых) с $\mathrm{E} X_{n}^{2}<\infty(n \in \mathbb{N})$ и таких, что $B_{n}=\mathrm{E} S_{n}^{2} \rightarrow \infty(n \rightarrow \infty)$, то, как показано в [1], для любой функции $\varphi(t) \nearrow \infty$ при $t \rightarrow \infty$

$$
\limsup _{n \rightarrow \infty} \frac{S_{n}}{\sqrt{B_{n}} \varphi\left(B_{n}\right)}=1 \text { п.н. }
$$

тогда и только тогда, когда

$$
\liminf _{n \rightarrow \infty} \frac{\varphi(n)}{\sqrt{2 \ln \ln n}}=1
$$

Назовем универсальной (для класса стандартных последовательностей случайных величин) такую нормировку, т.е. последовательность положительных чисел $\varphi(n) \nearrow \infty(n \rightarrow \infty)$, что для любой стандартной последовательности $\left\{X_{n}\right\}_{n \geqslant 1}$ п.н. выполняются соотношения

$$
\limsup _{n \rightarrow \infty} \frac{S_{n}}{\sqrt{n} \varphi(n)}=1 \text { и } \liminf _{n \rightarrow \infty} \frac{S_{n}}{\sqrt{n} \varphi(n)}=-1
$$

Теорема. Свойство универсальности нормировки $\{\varphi(n)\}_{n \geqslant 1}$ равносильно справедливости условия (2).

Необходимость утверждения следует из приведенного выше резултата [1]. Кроме того, последовательность $\left\{-X_{n}\right\}_{n \geqslant 1}$ будет стандартной, если стандартна $\left\{X_{n}\right\}_{n \geqslant 1}$. Поэтому достаточно доказать первое соотношение (3) для любой нормировки $\{\varphi(n)\}_{n \geqslant 1}$, удовлетворяющей (2).

Построим вначале вспомогательую последовательность $\{\psi(n)\}_{n \geqslant 1}$. Положим $L L(n)=$ $\ln \ln n$ при $n \geqslant 3$ и $L L(1)=L L(2)=1$. Возьмем две возрастающие последовательности натуральных чисел $\left\{A_{k}\right\}_{k \geqslant 1}$ и $\left\{B_{k}\right\}_{k \geqslant 1}$ такие, что $A_{1}=1, A_{k}<B_{k}<A_{k+1}-1$ при всех $k \in \mathbb{N}$. Пусть также

$$
\sum_{k=1}^{\infty} \frac{1}{L L\left(A_{k}\right)}<\infty \text { и } \sum_{k=1}^{\infty} \sum_{n \in\left(B_{k}, A_{k+1}\right)} \frac{1}{n \ln n}=\infty .
$$

Нетрудно видеть, что такие последовательности существуют, причем $\left(B_{k}, A_{k+1}\right) \cap \mathbb{N} \neq \varnothing$ при любом $k \in \mathbb{N}$. Определим

$$
\psi(n)=\sqrt{2 L L\left(B_{k}\right)} \text { для } n \in\left[A_{k}, B_{k}\right] \text { и } \psi(n)=\sqrt{2 L L(n)} \text { для } n \in\left(B_{k}, A_{k+1}\right), k \in \mathbb{N} .
$$

Работа вьполнена при поддержке Российского фонда фундаментальных исследований (гранты №№ 00-01-00131) и фоонда INTAS (грант № 99-01317). 
Лемма. Данная последовательность $\{\psi(n)\}_{n \geqslant 1}$ есть универсальная нормировка.

Доказательство леммы состоит в том, чтобы для каждого $C>3$ установить соотношение

$$
\limsup _{k \rightarrow \infty} \frac{S_{m_{k}}}{\sqrt{m_{k}} \psi\left(m_{k}\right)} \geqslant \sqrt{\frac{C-1}{C}}-\sqrt{\frac{1}{C}},
$$

где $m_{k}=\sum_{j=1}^{k} n_{j}, n_{j}=\left[C^{j}\right], k, j \in \mathbb{N}$. Для этого проверяем, что

$$
\limsup _{k \rightarrow \infty} \frac{S m_{k}-S m_{k-1}}{\sqrt{n_{k}} \psi\left(n_{k}\right)} \geqslant 1 \text { п.н. }
$$

Здесь используется резултат [2], согласно которому

$$
\sum_{k=1}^{\infty} \mathrm{P}\left(S_{n_{k}}>\psi\left(n_{k}\right) \sqrt{n_{k}}\right)<\infty \Longleftrightarrow \sum_{k=1}^{\infty}\left(\psi\left(n_{k}\right)\right)^{-1} \exp \left\{-\frac{\psi^{2}\left(n_{k}\right)}{2 \sigma^{2}\left(n_{k}\right)}\right\}<\infty,
$$

где $\sigma^{2}(n)$ - это дисперсия урезанной величины $X_{n} I\left\{\left|X_{n}\right|<\sqrt{n}\right\}$.

Теперь введем множество $\mathscr{K}_{C}$, состоящее из натуральных $k$, для которых на $\left[n_{k}+1, m_{k}\right]$ найдется хотя бы одна точка последовательности $\left\{A_{n}\right\}_{n \geqslant 1}$. Пользуясь тем, что промежутки $\left[n_{k}+1, m_{k}\right] \subset\left[C^{k}, C^{k+1} /(C-1)\right]$ (и тем самьм не пересекаются при разных $k$ ), а также условием (4), леммой Бореля-Кантелли и неравенством Чебышёва, выводим, что в случае бесконечного множества $\mathscr{K}_{C}$

$$
\lim _{k \rightarrow \infty, k \in \mathscr{K}_{C}} \frac{S_{m_{k}}-S_{m_{k-1}}}{\sqrt{m_{k}} \psi\left(n_{k}\right)}=0 \text { п.н. }
$$

Поскольку $m_{k} / n_{k} \sim C /(C-1)$ при $k \rightarrow \infty$, из (7) и (8) вытекает, что при каждом $C>3$ и любой структуре множества $\mathscr{K}_{C}$

$$
\limsup _{k \rightarrow \infty, k \notin \mathscr{K}_{C}} \frac{S_{m_{k}}}{\sqrt{m_{k}} \psi\left(n_{k}\right)} \geqslant \sqrt{\frac{C-1}{C}} \text { п.н. }
$$

Из (9) и теоремы Хартмана-Винтнера, а также соотношения $\lim _{k \rightarrow \infty, k \notin \mathscr{K}_{C}} \psi\left(m_{k}\right) / \psi\left(n_{k}\right)=1$ следует (6), что завершает доказательство леммы.

Продолжим доказательство теоремы. С учетом (2) достаточно рассматривать лишь такие нормировки, что $\varphi(n) \geqslant \sqrt{2 L L(n)}$ при всех $n \in \mathbb{N}$. В силу (2) найдется последовательность натуральных чисел $1=j_{1}<j_{2}<\cdots$, удовлетворяющих условиям

$$
\lim _{k \rightarrow \infty} \frac{\varphi\left(j_{k}\right)}{\sqrt{2 L L\left(j_{k}\right)}}=1, \quad \sum_{k=1}^{\infty} \frac{1}{L L\left(j_{k}\right)}<\infty \text { и } L L\left(j_{k}\right)-L L\left(j_{k-1}\right) \geqslant 2
$$

при всех $k \geqslant 2$. Введем $\beta(1)=\varphi(1)$ и $\beta(n)=\varphi\left(j_{k}\right)$ для $n \in\left(j_{k-1}, j_{k}\right], k \geqslant 2$. Тогда $\varphi(n) \leqslant \beta(n)$ при всех $n \in \mathbb{N}$. Положим $A_{1}=1, B_{1}=2, A_{2}=4, B_{2}=5$ и для $k \geqslant 3$ пусть

$$
A_{k}=j_{k-1}+1, \quad B_{k}=\max \left\{n \in\left(j_{k-1}+1, j_{k}\right) \cap \mathbb{N}: \sum_{m \in\left(n, j_{k}\right]} \frac{1}{m \ln m} \geqslant 1\right\} .
$$

По этим $\left\{A_{k}\right\}_{k \geqslant 1}$ и $\left\{B_{k}\right\}_{k \geqslant 1}$ определим $\{\psi(n)\}_{n \geqslant 1}$ согласно (5). В силу наших построений $\beta(n) \sim \psi(n)$ при $n \rightarrow \infty$. Поэтому утверждение теоремы вытекает из доказанной леммы.

Тем самьм получено обобщение теоремы Хартмана-Винтнера, а также результата недавней работы [3]. В частности, дается положительный ответ на вопрос, поставленный в [3], о существовании универсальной нормировки, для которой $\lim \sup _{n \rightarrow \infty} \varphi(n) / \sqrt{2 \ln \ln n}=\infty$.

\section{СПИСОК ЛИТЕРАТУРЫ}

[1] А. В. Булинский // Теория вероятн. и ее примен. 1977. Т. 22. № 2. С. 407-409. [2] C. C. Heyde // Z. Wahrscheinlichkeitstheor. Verw. Geb. 1969. V. 11. P. 181-192. [3] O. Klesov, A. Rosalsky // Stochastic Anal. Appl. 2001. V. 19. №4. P. 627-641.

Московский государственный

Принято редколлегией университет им. М.В. Ломоносова 23.01 .2002 